\title{
Flavor Changing Neutral Higgs Bosons Meet the Top and the Tau at Hadron Colliders
}

\author{
Chung Kao* \\ Homer L. Dodge Department of Physics and Astronomy, University of Oklahoma, Norman, OK \\ 73019, USA \\ E-mail: Chung.Kao@ou.edu
}

\begin{abstract}
We employ a general two Higgs doublet model to investigate the discovery potential of flavor changing neutral Higgs (FCNH) interactions for a Higgs boson decays into leptons in pp collisions, $p p \rightarrow \phi^{0} \rightarrow \tau^{\mp} \mu^{ \pm}+X$ from gluon fusion, where $\phi^{0}$ could be a CP-even scalar $\left(h^{0}, H^{0}\right)$ or a CP-odd pseudoscalar $\left(A^{0}\right)$. The light Higgs boson $h^{0}$ is found to resemble closely the standard Higgs boson at the Large Hadron Collider (LHC). In the alignment limit of $\cos (\beta-\alpha) \cong 0$, for $h^{0}-H^{0}$ mixing, FCNH couplings of $h^{0}$ are naturally suppressed, but such couplings of the heavier Higgs bosons $\left(H^{0}, A^{0}\right)$ are sustained by $\sin (\beta-\alpha) \simeq 1$. We evaluate physics backgrounds from dominant processes with realistic acceptance cuts and tagging efficiencies. We find promising results for the LHC with $\sqrt{s}=14 \mathrm{TeV}$, and future pp colliders with $\sqrt{s}=27 \mathrm{TeV}$ and $100 \mathrm{TeV}$.
\end{abstract}

European Physical Society Conference on High Energy Physics - EPS-HEP2019 -

10-17 July, 2019

Ghent, Belgium

\footnotetext{
* Speaker.

${ }^{\dagger}$ This article is base on a recent paper published in Phys. Lett. B. [1]
} 


\section{Introduction}

Recent studies at the CERN Large Hadron Collider (LHC) by ATLAS and CMS experiments confirm that the properties of the discovered Higgs boson are in good agreement with the expectations from the Standard Model (SM) Higgs boson [2, 3]. The most important experimental goals of the LHC and future high energy colliders are to test the Standard Model with precision and to search for new physics beyond the Standard Model.

There has been a lot of interest in the flavor changing neutral Higgs $(\mathrm{FCNH})$ interaction among leptons at the LHC. There was a $2.4 \sigma$ excess of $h^{0} \rightarrow \tau \mu$ above the background in CMS Run 1 data, with the best fit branching fraction [4] $\mathscr{B}\left(h^{0} \rightarrow \tau \mu\right) \simeq(0.84 \pm 0.38) \%$, which is consistent with ATLAS Run 1 result [5] of $\mathscr{B}\left(h^{0} \rightarrow \tau \mu\right) \simeq(0.77 \pm 0.62) \%$. But the excess was ruled out by 2016 CMS data [6], with upper limit $B\left(h^{0} \rightarrow \tau \mu\right) \lesssim 0.25 \%$.

Our investigation was motivated by the CMS experimental $2 \sigma$ hint for $h^{0} \rightarrow \tau \mu$ [4]. While it has subsequently disappeared [6], it in fact motivates further the search for $H^{0}, A^{0} \rightarrow \tau \mu$, involving heavy Higgs bosons, as we shall explain. Note in particular that, in face of the current semileptonic anomalies in B decays, a general two Higgs doublet model (g2HDM) had been invoked [7] over the disfavored conventional Type II of two Higgs doublet models (2HDM-II) [8]. While the situation with the anomalies are as yet inconclusive, we adopt the g2HDM without the usual $Z_{2}$ symmetry to forbid flavor changing neutral Higgs couplings [9]. The alignment [10] of two Higgs doublets may be at work instead of $Z_{2}$ or Natural Flavor Conservation [9]. Removing interactions of the extra scalars with vector boson pairs $\left(H^{0} W W\right.$ and $\left.H^{0} Z Z\right)$, other than the SM-Higgs, is known as the alignment limit $[11,12,13]$.

In SM, $h^{0} \rightarrow \tau \mu$ is highly suppressed, but in g2HDM without any $Z_{2}$ symmetry, this decay is in principle possible at tree level. We adopt the following interaction Lagrangian [14, 15],

$$
\begin{gathered}
\frac{-1}{\sqrt{2}} \sum_{F=U, D, E} \bar{F}\left\{\left[\kappa^{F} s_{\beta-\alpha}+\rho^{F} c_{\beta-\alpha}\right] h^{0}+\left[\kappa^{F} c_{\beta-\alpha}-\rho^{F} s_{\beta-\alpha}\right] H^{0}-i \operatorname{sgn}\left(Q_{F}\right) \rho^{F} A^{0}\right\} P_{R} F \\
-\bar{U}\left[V \rho^{D} P_{R}-\rho^{U \dagger} V P_{L}\right] D H^{+}-\bar{v}\left[\rho^{E} P_{R}\right] E H^{+}+\text {H.c. }
\end{gathered}
$$

where $P_{L}, P_{R} \equiv\left(1 \mp \gamma_{5}\right) / 2, c_{\beta-\alpha}=\cos (\beta-\alpha), s_{\beta-\alpha}=\sin (\beta-\alpha), \tan \beta \equiv v_{2} / v_{1}$, and $\alpha$ is the mixing angle between neutral Higgs scalars [8]. The $\kappa$ matrices are diagonal and fixed by fermion masses, $\kappa^{F}=\sqrt{2} m_{F} / v$ with $v \simeq 246 \mathrm{GeV}$, while $\rho$ matrices are in general not diagonal. The off diagonal elements of $\rho$ are tree level FCNH couplings.

The recent CMS data gives the bound on FCNH coupling [6] $\sqrt{\left|Y_{\tau \mu}\right|^{2}+\left|Y_{\mu \tau}\right|^{2}}=\tilde{\rho}_{\tau \mu}\left|c_{\beta-\alpha}\right|<$ $1.43 \times 10^{-3}$, where $\tilde{\rho}_{\tau \mu} \equiv \sqrt{\left(\left|\rho_{\tau \mu}\right|^{2}+\left|\rho_{\mu \tau}\right|^{2}\right) / 2}$. However, $Y_{\tau \mu}=\rho_{\tau \mu} c_{\beta-\alpha} / \sqrt{2}$ may be small because of alignment $[11,12,13]$ with $c_{\beta-\alpha} \rightarrow 0$. The leptonic FCNH Yukawa couplings of the heavy $H^{0}$ boson, $Y_{H \tau \mu}=-\rho_{\tau \mu} s_{\beta-\alpha} / \sqrt{2}$ would approach the $A^{0} \mathrm{FCNH}$ coupling $Y_{A \tau \mu}=-\rho_{\tau \mu} / \sqrt{2}$ in the alignment limit, since $s_{\beta-\alpha} \rightarrow 1$. While the recent CMS limit implies $B\left(h^{0} \rightarrow \tau \mu\right)$ must be small, $B\left(H^{0} \rightarrow \tau \mu\right)$ and $B\left(A^{0} \rightarrow \tau \mu\right)$ can still be sizable and should be probed experimentally.

In this article, we study the discovery potential for the decays $H^{0}, A^{0} \rightarrow \tau^{ \pm} \mu^{\mp}$, followed by $\tau$ decays into an electron and neutrinos or into a $\tau$-jet $\left(\pi, \rho\right.$, or $\left.a_{1}\right)$ and neutrino. Some promising results are presented for 14 and $27 \mathrm{TeV}$ center of mass $(\mathrm{CM})$ energies for an integrated luminosity $\mathscr{L}$ $=300$ and $3000 \mathrm{fb}^{-1}$, in sync with future High Luminosity (HL) and High Energy (HE) LHC [16]. 


\section{Constraints on Relevant Parameters}

To evaluate the production rate of $p p \rightarrow \phi^{0} \rightarrow \tau \mu+X, \phi^{0}=H^{0}, A^{0}$, the most relevant parameters are $\rho_{\tau \mu}, \rho_{\mu \tau}$ for the decay $\phi^{0} \rightarrow \tau \mu$, and $\rho_{t t}$ for the production $g g \rightarrow \phi^{0}$ via the top loop. A potentially large $\rho_{t c}$ induces [17] $\phi^{0} \rightarrow t \bar{c}, \bar{t} c$, which can dilute the $H^{0} / A^{0} \rightarrow \tau \mu$ branching ratio, while $\rho_{c t}$ is subject to tight constraints by $B$ physics data: $\rho_{c t}<0.1$ [17].

The LHC data favor the alignment limit with $|\cos (\beta-\alpha)| \ll 1$. We take $\cos (\beta-\alpha)=0.1$ for illustration, although larger values are allowed in the general 2HDM [17, 18]. As for other $\rho$ matrix elements, we set $\rho_{f f}=\kappa_{f}=\sqrt{2} m_{f} / v$ for diagonal elements except $\rho_{t t}$, and ignore offdiagonal ones except $\rho_{\tau \mu}, \rho_{\mu \tau}$ and $\rho_{t c}$. Degenerate extra scalar masses, i.e. $M_{H^{0}}=M_{A^{0}}=M_{H^{ \pm}}$, is assumed for simplicity. In general $\rho_{t t}$ is complex and it may contribute to CP violation and Baryogenesis [19]. For simplicity, we will take it to be real in this work.

Constraints on $\rho_{\tau \mu}$ and $\rho_{\mu \tau}$ by various low-energy processes containing tau and muon are discussed in the literature [20, 21, 22, 23]. It is found that $\tau \rightarrow \mu \gamma$ is most relevant.

The FCNH coupling $\rho_{t c}$ induces $t \rightarrow c h$ decay [24, 25], and the recent ATLAS limit [26] of

$$
\mathscr{B}\left(t \rightarrow c h^{0}\right)<1.1 \times 10^{-3}(95 \% \text { C.L. })
$$

directly constrains $\rho_{t c}$ if $c_{\beta-\alpha}$ is nonzero. Let us define $\tilde{\rho}_{t c}=\sqrt{\left|\rho_{t c}\right|^{2}+\left|\rho_{c t}\right|^{2}} / \sqrt{2}$ as a convenient FCNH coupling [17, 27]. Combining experimental limits from LHC Higgs data and $B$ physics, we consider $\rho_{\tau \mu}=\rho_{\mu \tau}<0.01$, and $\left|\tilde{\rho}_{t c} c_{\beta-\alpha}\right|=\lambda_{t c h} \lesssim 0.064$ [26]. To be consistent with $B$ physics constraints, we choose

$$
\rho_{t t}=0.2 \times\left(M_{\phi} / 150 \mathrm{GeV}\right)
$$

for $\phi^{0}=H^{0}$ or $A^{0}$, which always satisfies the $b \rightarrow s \gamma$ constraint for the heavy Higgs scalar mass considered in our study.

\section{Higgs Signal and Physics Background}

In this section, we discuss the prospect of discovering FCNH interactions from heavy Higgs bosons $H^{0}$ and $A^{0}$ decaying into $\tau^{ \pm} \mu^{\mp}$. The ATLAS and CMS experimental constraints suggest that $c_{\beta-\alpha}$ must be very small to be consistent with the alignment limit. We will choose $c_{\beta-\alpha}=0.1$ for case studies.

\subsection{The Higgs Potential and Decay Final States}

For the heavy CP-even $H^{0}$ boson, the most important SM decay channels are $b \bar{b}, t \bar{t}, W W$, and $Z Z$. In addition, $t c$ and $h^{0} h^{0}$ channels might become dominant in some regions of parameter space. The CP-odd pseudoscalar $A^{0}$ boson has significant decays into $b \bar{b}, t \bar{t}$, as well as possible dominant contributions from $t c$ and $Z h^{0}$ channels.

To study heavy boson $H^{0}$ or $A^{0}$ decays involving the light Higgs boson $h^{0}$, let us consider a general CP-conserving Higgs potential [11]

$$
\begin{aligned}
V & \left.=m_{11}^{2}\left|\Phi_{1}\right|^{2}+m_{22}^{2}\left|\Phi_{2}\right|^{2}-\left[m_{12}^{2} \Phi_{1}^{\dagger} \Phi_{2}+\text { h.c. }\right]+\frac{1}{2} \lambda_{1}\left|\Phi_{1}\right|^{4}+\frac{1}{2} \lambda_{2} \mid \Phi_{2}\right)\left.\right|^{4}+\lambda_{3}\left|\Phi_{1}\right|^{2}\left|\Phi_{2}\right|^{2} \\
& +\lambda_{4}\left(\Phi_{1}^{\dagger} \Phi_{2}\right)\left(\Phi_{2}^{\dagger} \Phi_{1}\right)+\left[\frac{1}{2} \lambda_{5}\left(\Phi_{1}^{\dagger} \Phi_{2}\right)^{2}+\lambda_{6}\left|\Phi_{1}\right|^{2}\left(\Phi_{1}^{\dagger} \Phi_{2}\right)+\lambda_{7}\left|\Phi_{2}\right|^{2}\left(\Phi_{1}^{\dagger} \Phi_{2}\right)+\text { h.c. }\right]
\end{aligned}
$$


Applying minimization conditions, we can express the triple Higgs coupling $g_{H h}$ in terms of physical masses and mixing angles $[11,28]$

$$
g_{H h h} \simeq-\frac{c_{\beta-\alpha}}{v}\left[4 m_{A}^{2}-2 m_{h}^{2}-m_{H}^{2}+4 \lambda_{5} v^{2}+\frac{2 v^{2}}{\tan 2 \beta}\left(\lambda_{6}-\lambda_{7}\right)+\frac{2 v^{2}}{\sin 2 \beta}\left(\lambda_{6}+\lambda_{7}\right)\right] .
$$

For simplicity, we take the heavy Higgs states $H^{0}, A^{0}$ and $H^{ \pm}$to be degenerate and set $\lambda_{6,7}=0$. We observe that for a fixed value of $\tan \beta$, increasing $\lambda_{5}$ from -1 to 0 lowers the cross section of $p p \rightarrow H^{0} \rightarrow \tau \mu+X$ while increasing the trilinear Higgs coupling, $g_{H h h}$, which enhances the branching fraction of $H^{0} \rightarrow h^{0} h^{0}$,

For the $H^{0}$ boson, $\tilde{\rho}_{t c}, \lambda_{5}$ and $\tan \beta$ play crucial roles in affecting the $H^{0} \rightarrow \tau \mu$ branching ratio. We use 2HDMC [29] to scan over $150 \mathrm{GeV} \leq M_{H} \leq 500 \mathrm{GeV}$ and $1 \leq \tan \beta \leq 10$ for $\lambda_{5}=0$. For $M_{H}>2 m_{t}, H^{0} \rightarrow t \bar{t}, h^{0} h^{0}$ and $t c$ channels might become significant.

The pseudoscalar $A^{0}$ decays mostly into fermions. Only $\rho_{t c}$ has significant impact on the branching fractions. For $\tilde{\rho}_{t c} \gtrsim 0.5, A^{0} \rightarrow t \bar{c}+\bar{t} c$ becomes dominant. Furthermore, for $M_{A}>220$ $\mathrm{GeV}, A^{0} \rightarrow Z h^{0}$ also makes significant contribution. For $M_{A}>2 m_{t}$, the $t \bar{t}$ channel starts to dominate.

\subsection{Higgs Signal}

Our main signal channel is the production and FCNH decay of a heavy Higgs boson $\left(\phi^{0}=\right.$ $H^{0}, A^{0}$ ) via gluon fusion, $p p \rightarrow \phi^{0} \rightarrow \tau \mu+X[30,31,32,33,34,35,36]$. With the $\tau$ decaying leptonically, we are looking for a final state of two opposite sign leptons and missing energy. With a hadronically decaying $\tau$, a final state with a $\tau$-jet $\left(j_{\tau}\right)$, a muon, and missing energy is needed. We have evaluated the FCNH signal cross sections with analytic matrix element and leading order CT14 parton distribution functions $[38,37]$. To include higher order corrections we calculate Kfactors with Higlu [39] for $p p \rightarrow \phi^{0}+X$.

\subsection{Standard Model Backgrounds}

The dominant background for leptonic final states comes from production of $\tau^{+} \tau^{-}$and , $W^{+} W^{-}$. For hadronic channel, we have considered $p p \rightarrow W^{ \pm} j \rightarrow \mu j+\mathbb{E}_{T}+X$ as the most dominant background along with the $\tau \tau$ channel. For hadronic channel, $t \bar{t}$ contribution is highly suppressed, when we veto any event with more than one b jet, with $p_{T}>20 \mathrm{GeV}$ and $|\eta|<4.7$. We have used MADGRAPH [40] and HELAS [41] to generate matrix elements for the backgrounds.

\subsection{Realistic acceptance cuts}

To study the discovery potential for the FCNH signal, we apply realistic acceptance cuts proposed by CMS $[4,6]$ at $\sqrt{s}=13 \mathrm{TeV}$. In addition, we apply Gaussian smearing for particle momenta to simulate detector effects based on ATLAS and CMS specifications.

We note that, as the Higgs boson mass increases, $M_{\mathrm{col}}(\tau \mu)$ cut becomes more effective. For leptonic channel, $p p \rightarrow W^{+} W^{-}+X$ becomes more dominant than $p p \rightarrow \tau \tau+X$.

\section{Discovery Potential}

To estimate the discovery potential, we require that the lower limit on the signal plus background should be larger than the corresponding upper limit on the background with statistical 
fluctuations, which leads to [42]

$$
\sigma_{S} \geq \frac{N}{\mathscr{L}}\left[N+2 \sqrt{\mathscr{L} \sigma_{B}}\right]
$$

where $\sigma_{S}$ and $\sigma_{B}$ are the signal and background cross sections, respectively, and $\mathscr{L}$ is the integrated luminosity. Choosing $N=2.5$, we obtain a $5 \sigma$ significance.
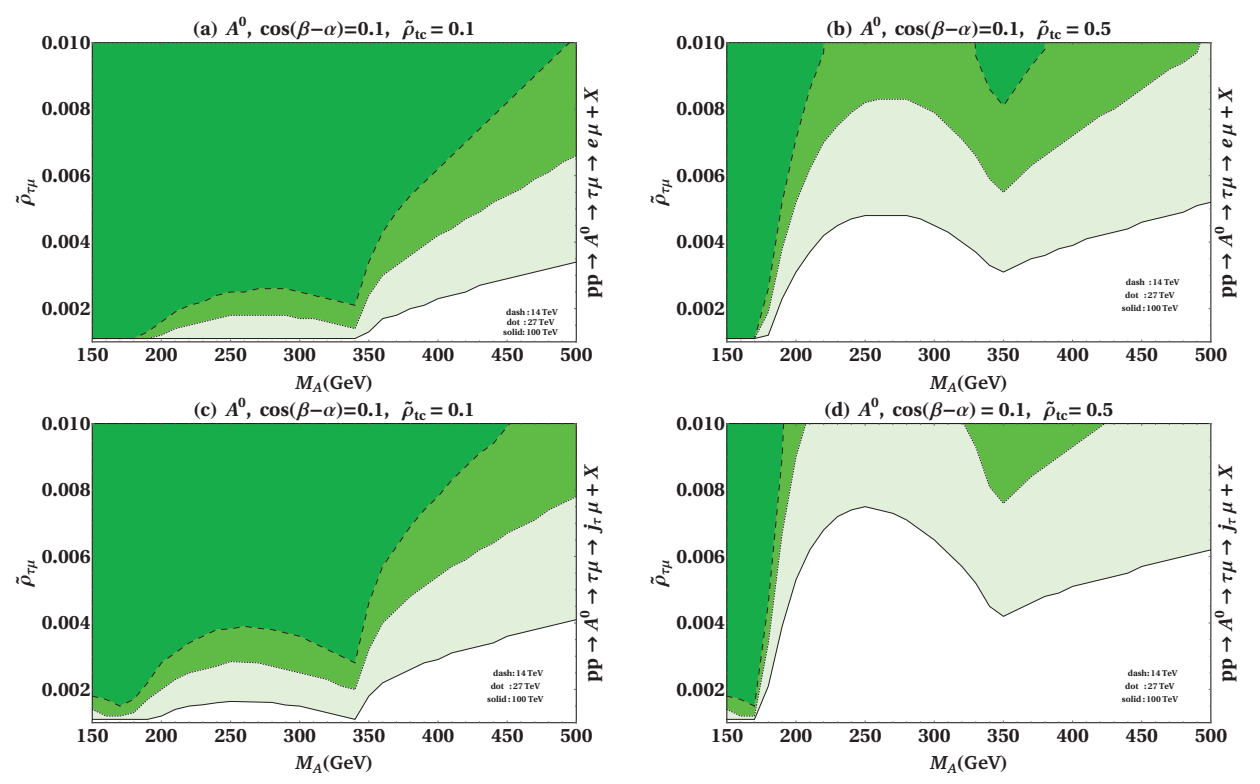

Figure 1: Discovery range at the LHC and future hadron colliders with $\sqrt{s}=14 \mathrm{TeV}$ (green dark shading), $27 \mathrm{TeV}$ (intermediate shading) and $100 \mathrm{TeV}$ (light shading) for $p p \rightarrow A^{0} \rightarrow \tau \mu+X$ in the $\left(M_{A}, \tilde{\rho}_{\tau \mu}\right)$ plane. We require $5 \sigma$ significance for $3000 \mathrm{fb}^{-1}$. Top (bottom) row is for leptonic (hadronic) tau decay for $\tilde{\rho}_{t c}=$ $0.1[(\mathrm{a})$ and $(\mathrm{c})]$ and $\tilde{\rho}_{t c}=0.5[(\mathrm{~b})$ and $(\mathrm{d})]$.

\subsection{Discovery Reach for Pseudoscalar $A^{0}$}

The pseudoscalar $A^{0}$ has higher production cross section than $H^{0}$, and with no suppression coming from $A^{0} \rightarrow h^{0} h^{0}$. Fig. 1 shows the discovery region for $p p \rightarrow A^{0} \rightarrow \tau \mu+X$ in the $\left(M_{A}, \tilde{\rho}_{\tau \mu}\right)$ plane, for $\tilde{\rho}_{t c}=0.1$ and 0.5 . Because of high QCD backgrounds, performance for hadronic $\tau$ decay is worse than leptonic decay, despite its higher branching ratios.

We show our results for $\sqrt{s}=14,27$ and $100 \mathrm{TeV}$. At low masses with $M_{A}<180 \mathrm{GeV}$, the entire range of $\rho_{\tau \mu}$ is detectable for $3000 \mathrm{fb}^{-1}$. For an intermediate range $\left(200 \mathrm{GeV}<M_{A}<\right.$ $300 \mathrm{GeV}$ ), our discovery region starts shrinking because of $A^{0} \rightarrow t \bar{c}$ as shown in Fig. 1. For higher mass range $\left(M_{A}>300 \mathrm{GeV}\right)$, we see a slight increase in the $5 \sigma$ region before and around $M_{A} \sim 2 m_{t}$, owing to the rise in production cross section for $g g \rightarrow A^{0}$. The $A^{0} \rightarrow t \bar{t}$ decay suppresses our signal towards higher masses beyond $M_{A} \gtrsim 360 \mathrm{GeV}$.

\subsection{Discovery Reach for Heavy CP-even Scalar $H^{0}$}

For the heavy CP-even boson $H^{0}$, the situation is quite different. The branching fraction for $H^{0} \rightarrow \tau \mu$ is very sensitive to $\rho_{t c}, \tan \beta$ and $\lambda_{5}$, that can affect the $H^{0} \rightarrow t \bar{c}+\bar{t} c$ and $h^{0} h^{0}$ decays. In 
(a) $\sqrt{s}=14 \mathrm{TeV}, M_{H}=300 \mathrm{GeV}, \quad \tilde{\rho}_{\mathrm{tc}}=0.1$

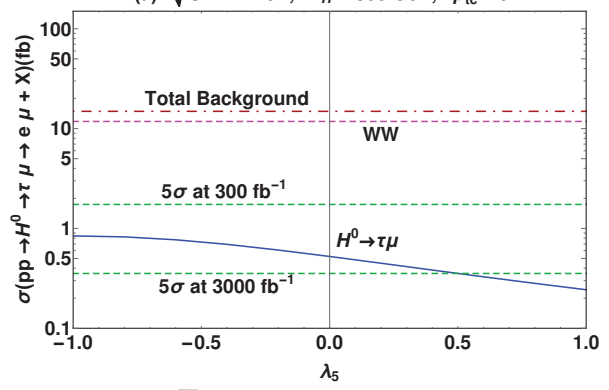

(c) $\sqrt{s}=100 \mathrm{TeV}, M_{H}=300 \mathrm{GeV}, \quad \tilde{\rho}_{\mathrm{tc}}=0.1$

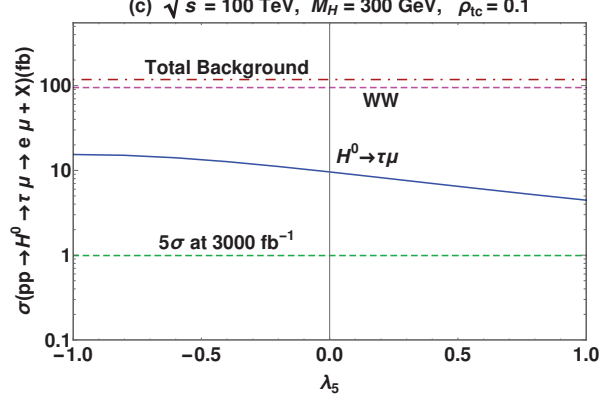

(b) $\sqrt{s}=27 \mathrm{TeV}, M_{H}=300 \mathrm{GeV}, \quad \tilde{\rho}_{\mathrm{tc}}=0.1$

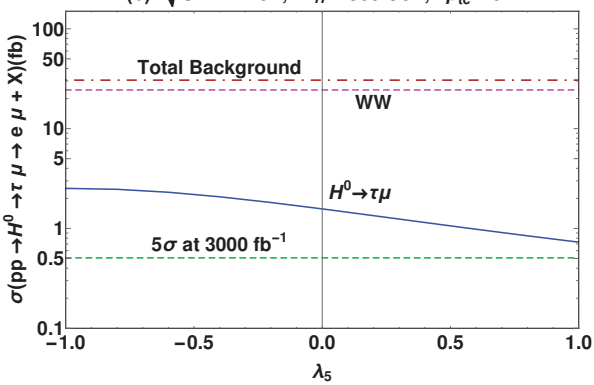

(d) $\sqrt{s}=100 \mathrm{TeV}, M_{H}=300 \mathrm{GeV}, \quad \tilde{\rho}_{\mathrm{tc}}=0.5$

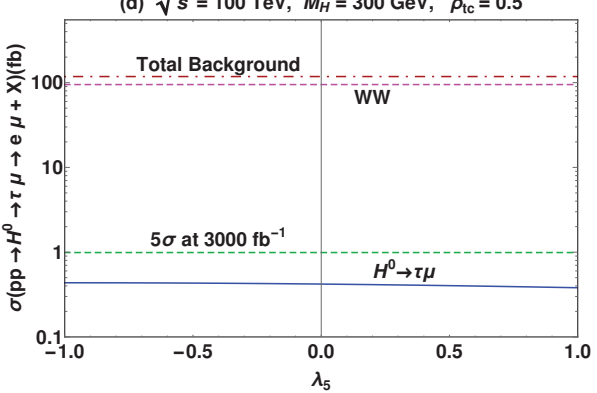

Figure 2: Cross section (in fb) of $p p \rightarrow H^{0} \rightarrow \tau \mu \rightarrow e \mu+X$ (blue solid) at (a) $\sqrt{s}=14 \mathrm{TeV}$, (b) $27 \mathrm{GeV}$, and (c) $100 \mathrm{TeV}$, as a function of $\lambda_{5}$ with $M_{H}=300 \mathrm{GeV}, \rho_{\tau \mu}=0.01, \tan \beta=1, c_{\beta-\alpha}=0.1$, and $\tilde{\rho}_{t c}=0.1$. We also present the results for (d) $\tilde{\rho}_{t c}=0.5$ at $\sqrt{s}=100 \mathrm{TeV}$.

order to understand the effect of $\lambda_{5}$, we perform a case study for $p p \rightarrow H^{0} \rightarrow \tau \mu \rightarrow e \mu+X$ with $M_{H}=300 \mathrm{GeV}, \rho_{\tau \mu}=0.01$, and scan over $-1 \leq \lambda_{5} \leq 1$ for $\tan \beta=1$. Figure 2 shows cross section of $p p \rightarrow H^{0} \rightarrow \tau \mu \rightarrow e \mu+X$ for $\sqrt{s}=14,27$ and $100 \mathrm{TeV}$ and $\tilde{\rho}_{t c}=0.1$.

As a case study, let us choose the values of $\lambda_{5}=-1,0$, with $\tan \beta=1$ to preserve tree-level unitarity and stability for a general $2 \mathrm{HDM}$, which resembles the generic case with $0.001 \leq \rho_{\tau \mu} \leq$ 0.01 and $150 \mathrm{GeV} \leq M_{H} \leq 500 \mathrm{GeV}$. The discovery contours for $p p \rightarrow H^{0} \rightarrow \tau \mu+X$ are are shown in Fig. 3. There is a large discoverable region in the low mass regime $\left(M_{H}<180 \mathrm{GeV}\right)$. However, as we start increasing $M_{H}$, first $H^{0} \rightarrow t \bar{c}$, then $H^{0} \rightarrow h^{0} h^{0}$, then $H^{0} \rightarrow t \bar{t}$ become dominant. The likelihood of detection increases as we reduce the value of $\lambda_{5}$, from 0 to -1 .

\section{Conclusion}

The general two Higgs doublet model offers a very rich phenomenology for flavor changing neutral Higgs interactions with fermions. In the general 2HDM, the coupling probed is $\lambda_{h \tau \mu}=$ $\rho_{\tau \mu} \cos (\beta-\alpha)$, which is expected to be small in the alignment limit of $\cos (\beta-\alpha) \rightarrow 0$, where the light CP-even Higgs boson $h^{0}$ approaches the standard Higgs boson.

For heavy Higgs states, the pseudoscalar $A^{0}$ boson has FCNH coupling $\lambda_{A \tau \mu}=\rho_{\tau \mu}$ that is independent of $\cos (\beta-\alpha)$, while the heavy CP-even scalar $H^{0}$ has FCNH coupling $\lambda_{H \tau \mu}=$ $\rho_{\tau \mu} \sin (\beta-\alpha)$, where $\sin (\beta-\alpha)$ is expected to be close to unity. Thus, they offer great promise to discover FCNH signals with lepton flavor violating production of $p p \rightarrow H^{0}, A^{0} \rightarrow \tau \mu+X$ at the LHC and future hadron colliders. 

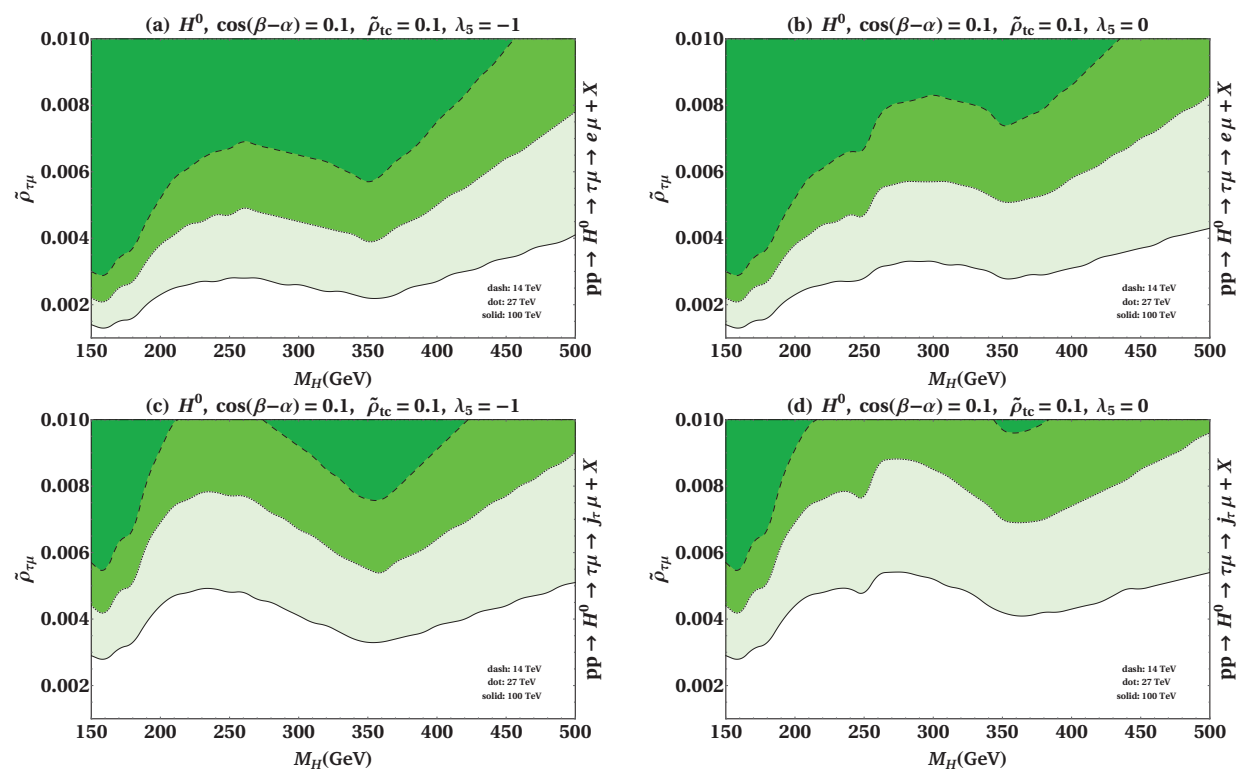

Figure 3: Discovery regions at the LHC and future hadron colliders with the $\sqrt{s}=14 \mathrm{TeV}$ (green dark shading), $27 \mathrm{TeV}$ (intermediate shading) and $100 \mathrm{TeV}$ (light shading) for $p p \rightarrow H^{0} \rightarrow \tau \mu+X$ in the $\left(M_{H}, \tilde{\rho}_{\tau \mu}\right)$ plane. We require at least $5 \sigma$ significance for $3000 \mathrm{fb}^{-1}$. Top (bottom) row is for leptonic (hadronic) tau decay with $\lambda_{5}=-1[(\mathrm{a})$ and (c) $]$ and $\lambda_{5}=0[(\mathrm{~b})$ and (d)].

We have investigated the prospects of discovering $H^{0}, A^{0} \rightarrow \tau \mu$ for the LHC and future high energy $p p$ colliders, and find promising results for LHC with $\cos (\beta-\alpha)=0.1, \tilde{\rho}_{t c}=0.1$, when $H^{0}, A^{0} \rightarrow t \bar{c}+\bar{t} c$ is not yet overwhelming for $M_{H}$ up to $300 \mathrm{GeV}$. It should be noted that $A^{0}$ is more promising than $H^{0}$ because of its higher production cross section and fewer decay channels affecting its decay to $\tau \mu$, but $H^{0}$ decay depends also on Higgs potential due to $h^{0} h^{0}$ mode. If $\tilde{\rho}_{t c}$ is considerably larger than $0.1, H^{0}, A^{0}$ decay to $t \bar{c}$ would suppress $\tau \mu$ observability, and a higher energy collider with a higher luminosity would be needed.

\section{Acknowledgements}

I am grateful to W.-S. Hou, R. Jain, M. Kohda, B. McCoy and A. Soni, for a very enjoyable collaboration.

\section{References}

[1] W. S. Hou, R. Jain, C. Kao, M. Kohda, B. McCoy and A. Soni, Phys. Lett. B 795, 371 (2019).

[2] A.M. Sirunyan et al. [CMS Collaboration], Eur. Phys. J. C 79, 421 (2019).

[3] The ATLAS Collaboration, ATLAS-CONF-2019-005.

[4] V. Khachatryan et al. [CMS Collaboration], Phys. Lett. B 749 (2015) 337.

[5] G. Aad et al. [ATLAS Collaboration], Eur. Phys. J. C 77, 70 (2017).

[6] A.M. Sirunyan et al. [CMS Collaboration], JHEP 1806, 001 (2018). 
[7] A. Crivellin, C. Greub and A. Kokulu, Phys. Rev. D 86, 054014 (2012).

[8] J.F. Gunion, H.E. Haber, G.L. Kane and S. Dawson, Front. Phys. 80, 1 (2000).

[9] S.L. Glashow and S. Weinberg, Phys. Rev. D 15, 1958 (1977).

[10] W.-S. Hou and M. Kikuchi, EPL 123, 11001 (2018).

[11] J.F. Gunion and H.E. Haber, Phys. Rev. D 67, 075019 (2003).

[12] M. Carena, I. Low, N.R. Shah and C.E.M. Wagner, JHEP 1404, 015 (2014).

[13] P.S. Bhupal Dev and A. Pilaftsis, JHEP 1412, 024 (2014).

[14] S. Davidson and H.E. Haber, Phys. Rev. D 72, 035004 (2005).

[15] F. Mahmoudi and O. Stål, Phys. Rev. D 81, 035016 (2010).

[16] W. Barletta, M. Battaglia, M. Klute, M. Mangano, S. Prestemon, L. Rossi and P. Skands, arXiv:1310.0290 [physics.acc-ph].

[17] B. Altunkaynak, W.-S. Hou, C. Kao, M. Kohda and B. McCoy, Phys. Lett. B 751, 135 (2015).

[18] W.-S. Hou, M. Kohda and T. Modak, Phys. Rev. D 98, 075007 (2018).

[19] K. Fuyuto, W.-S. Hou and E. Senaha, Phys. Lett. B 776, 402 (2018).

[20] S. Davidson and G.J. Grenier, Phys. Rev. D 81, 095016 (2010).

[21] D. Aristizabal Sierra and A. Vicente, Phys. Rev. D 90, 115004 (2014).

[22] I. Doršner, S. Fajfer, A. Greljo, J.F. Kamenik, N. Košnik and I. Nišandžić, JHEP 1506, 108 (2015).

[23] Y. Omura, E. Senaha and K. Tobe, Phys. Rev. D 94, 055019 (2016).

[24] W.-S. Hou, Phys. Lett. B 296, 179 (1992).

[25] K.-F. Chen, W.-S. Hou, C. Kao and M. Kohda, Phys. Lett. B 725, 378 (2013).

[26] M. Aaboud et al. [ATLAS Collaboration], JHEP 1905, 123 (2019).

[27] R. Jain and C. Kao, Phys. Rev. D 99, 055036 (2019).

[28] N. Craig, J. Galloway and S. Thomas, arXiv:1305.2424 [hep-ph].

[29] D. Eriksson, J. Rathsman and O. Stål, Comput. Phys. Commun. 181, 189 (2010).

[30] T. Han and D. Marfatia, Phys. Rev. Lett. 86, 1442 (2001).

[31] K.A. Assamagan, A. Deandrea and P.A. Delsart, Phys. Rev. D 67, 035001 (2003).

[32] R. Harnik, J. Kopp and J. Zupan, JHEP 1303, 026 (2013).

[33] M. Buschmann, J. Kopp, J. Liu and X.-P. Wang, JHEP 1606, 149 (2016).

[34] M. Sher and K. Thrasher, Phys. Rev. D 93, 055021 (2016).

[35] R. Primulando and P. Uttayarat, JHEP 1705, 055 (2017).

[36] A. Bednyakov and V. Rutberg, Mod. Phys. Lett. A 33, 1850152 (2018).

[37] J. Gao et al., Phys. Rev. D 89, 033009 (2014).

[38] S. Dulat et al., Phys. Rev. D 93, 033006 (2016).

[39] M. Spira, hep-ph/9510347; Nucl. Instrum. Meth. A 389, 357 (1997).

[40] J. Alwall, M. Herquet, F. Maltoni, O. Mattelaer and T. Stelzer, JHEP 1106, 128 (2011).

[41] K. Hagiwara, J. Kanzaki, Q. Li and K. Mawatari, Eur. Phys. J. C 56, 435 (2008).

[42] H. Baer, M. Bisset, C. Kao and X. Tata, Phys. Rev. D 46, 1067 (1992). 